\title{
Postural Control and Verbal and Visual Working Memory Correlates in Nonclinical Psychosis
}

\author{
Ivanka Ristanovic ${ }^{\mathrm{a}}$ K. Juston Osborne ${ }^{\mathrm{a}}$ Teresa Vargas $^{\mathrm{a}} \quad$ Tina Gupta $^{\mathrm{a}}$ \\ Vijay A. Mittal ${ }^{\mathrm{a}-\mathrm{c}}$ \\ ${ }^{a}$ Department of Psychology, Northwestern University, Evanston, IL, USA; ${ }^{b}$ Department of Psychiatry, Northwestern \\ University, Chicago, IL, USA; ' Institute for Policy Research, Northwestern University,, Evanston, IL, USA
}

\section{Keywords}

Nonclinical psychosis - Postural control · Cerebellarthalamic-cortical-cerebellar circuit - Working memory

\begin{abstract}
Introduction: Motor and cognitive abnormalities are well documented in psychosis spectrum disorders. Evidence suggests these deficits could be pronounced because of disruptions in the cerebellar-thalamic-cortical-cerebellar (CTCC) circuit, a network thought to be heavily implicated in motor and higher cognitive functioning. Although significant research has been done on this topic in individuals with schizophrenia and those at a clinical high risk for psychosis, much less is known about deficits at the lower end of the spectrum. Methods: In this study, we extended the understanding of motor abnormalities across the psychosis continuum by examining postural sway deficits in the nonclinical psychosis (NCP) population. Furthermore, we linked these deficits to verbal and visual working memory. High-NCP $(n=37)$ and low-NCP control $(n=31)$ participants completed an instrumental balance task, highly sensitive to subtle variations in postural sway, along with a brief working memory battery. Results: We found that high-NCP participants presented with increased postural sway area (i.e., worse postural con-
\end{abstract}

\section{KARGER}

(๑) 2019 S. Karger AG, Basel

karger@karger.com

www.karger.com/nps trol) relative to low-NCP controls on a difficult condition (with limited proprioceptive cues), but not on an easier condition. Furthermore, results indicated that the sway area was correlated with poorer performance on working memory tasks in the high-NCP group. Conclusion: These findings suggest that CTCC circuit abnormalities are present across the lower end of the psychosis spectrum and that they may be contributing to a range of motor and cognitive behaviors seen in the population. However, evidence suggests that the signs are subtle, and that sensitive assessment devices and challenging conditions may be necessary for detection.

(c) 2019 S. Karger AG, Basel

\section{Introduction}

An accumulating body of research suggests that psychotic experiences exist on a phenotypic continuum, from disorders such as schizophrenia at the severe end to subclinical levels of fleeting psychotic-like experiences at the lower end [1-3]. With respect to the latter group, in recent years we have come to understand that a sizable subset of the general population $(5-13 \% ;[1,2,4])$ reports nonclinical psychosis (NCP) symptoms. While these symptoms include experiences that characterize formal 
psychosis (e.g., unusual thoughts or hallucinations), they are brief, rare, and are not accompanied by distress or impairment. However, younger individuals reporting NCP symptoms are at an increased risk for converting to a psychotic disorder [5-8], indicating a shared vulnerability. Critically, NCP individuals present with similar signs of biological vulnerability (e.g., in areas such as cognitive function), albeit in an attenuated fashion [9-12]. Taken together with the high prevalence rate and the limited potential confounds characteristic of formal psychotic disorders [12], this shared continuum of vulnerability suggests that NCP may be a highly important construct for improving the understanding of psychosis.

Motor abnormalities such as neurological soft signs, abnormal involuntary movements, and catatonia are common in formal psychosis [13]. The movement domain also represents a particularly promising area for inquiry in NCP. First, select abnormal movement signs may be unique to psychosis risk domain [13-15], and this may be helpful in isolating a weaker signal. Second, our ability to detect subtle aberrations in motor behavior which, if present, are likely to characterize populations lower on the continuum, has dramatically improved in recent years due to the more widespread adoption of instrumental assessment modalities [16-20]. Third, there is preliminary evidence to suggest that some motor deficits have already been detected in NCP, including variable force $[12,18]$. Finally, and most critically, motor signs reflect abnormalities in brain circuits that are implicated in psychosis [18, 21-23]. Understanding these links, across a continuum, can yield important clues to improve the etiological understanding and to highlight new treatment targets. However, despite this promising work, to date, there has been limited research into motor function in this important area.

With respect to this last point, there is good preliminary evidence to suggest that motor behaviors linked to cerebellar-thalamic-cortical-cerebellar (CTCC) circuit dysfunction are implicated in psychosis. CTCC circuit function is critically implicated in conceptual models of schizophrenia $[24,25]$ and, thus far, related motor symptoms have been well recorded in populations at a clinical high risk (CHR) for transitioning to psychosis [16, 17, 21, 26] as well as in schizophrenia patients [19, 27-32]. Moreover, a recent study by our group demonstrated potential cerebellar deficits in the NCP population. Specifically, in a double-blind, crossover paradigm, NCP and control participants completed a motor-learning pursuit rotor task after cerebellar transcranial direct current stimulation at one visit, and after a sham (placebo) stimulation at the other visit. The results indicated that, following the sham condition, NCP participants showed significantly lower rates of motor learning across trials; after the active condition, they performed at comparable rates to controls [33]. However, while this provides some support for a potential continuum of cerebellar vulnerability, currently we lack studies of motor behavior that would serve to enrich this perspective.

We and several other groups of investigators have conducted studies utilizing a postural sway paradigm, and we now have good evidence to suggest that this is a sensitive measure of CTCC circuit dysfunction [17, 21, 27, 34]. Neuroimaging research implicates the CTCC circuit in postural control, indicating that the affected cerebellar networks are related to deficits in this specific motor domain across CHR and schizophrenia populations [21, 35]. Notably, a recent study by our group denoted the cerebellum as a distinct neural substrate in the pathogenesis of psychosis and found that decreased connectivity in the CTCC circuit was associated with a larger sway area in the CHR group relative to controls [21]. This finding was the most prominent in the most challenging postural sway condition where participants were asked to keep their eyes closed and feet together. Studies assessing individuals with schizophrenia [27, 36] and schizotypal personality disorder (SPD) [36] had similar results, supporting the consistent sway pattern across the psychosis spectrum. Taken together, these findings suggest that decreasing proprioceptive and visual cues increases the challenge to the cerebellum.

If the CTTC circuit does show vulnerability across the psychosis continuum, it will also be important to build a better understanding of cognitive correlates. As noted, procedural learning has already been implicated [33], but it is important to also consider that the CTCC circuit is implicated in modulating a variety of higher-order cognitive functions including working memory $[37,38]$. Correlates with sway area and cognitive performance in NCP will help to provide early building blocks for understanding how a common circuit dysfunction may contribute to a wide array of symptoms and characteristics, something that has been well established in formal psychosis with theories such as cognitive dysmetria $[24,25]$, but has not been investigated at the lower end of the psychosis continuum.

We aimed to examine postural sway as well as cognitive correlates in a group exhibiting high NCP and in lowNCP controls. We administered a task measuring discreet levels of postural sway across conditions designed to manipulate proprioceptive and visual cues and increasingly 
challenge the CTCC circuit. Based on our previous work with CHR youth, we hypothesized that, compared to the low-NCP group, the high-NCP group would show deficits in postural control in both easier (i.e., eyes open and feet apart condition) and more difficult (i.e., eyes closed and feet together) conditions. In addition, we examined the relationship between postural control and both visual and verbal working memory in the high-NCP group, and predicted that poorer postural control will be associated with poorer performance across these tasks.

\section{Materials and Methods}

\section{Participants}

Participants (mean age 19.12 years; range 18-26 years) were recruited from the Adolescent Development and Preventive Treatment Program (ADAPT) through an undergraduate research pool. Those who participated $(n=1,248)$ completed the LaunaySlade Hallucination Scale (LSHS) [39], and the opportunity to participate in the study was offered to those scoring in the top (highNCP) and bottom (low-NCP) 10th percentile ( $\geq 23$ or $\leq 3$, respectively). Out of those invited $(n=250), 72$ decided to participate. In an effort to limit sampling bias, the study description was not available during the research pool screening. Those participating were provided a detailed description of the study procedures during the consenting process. Four participants who did not complete the postural control task were excluded from all analyses. No other participants were excluded from the study. The final sample $(n=$ 68) consisted of 37 participants in the high-NCP group and 31 in the low-NCP control group. Antipsychotic treatment was an exclusion criterion, and no participants reported use of antipsychotics. In the entire sample, $7.4 \%$ reported taking antidepressants, $4.4 \%$ anti-anxiety medication, $23.5 \%$ birth control medication, and $13.2 \%$ other medications.

\section{Clinical Measures}

The LSHS is a well-validated [39] and commonly used scale for identifying NCP symptoms in healthy populations [9, 40,41]. It is a 12 -item self-report scale measuring hallucination proneness, with scores ranging from 0 to 48 . Participants were asked to rate hallucinatory experiences (e.g., "In my daydreams I can hear the sound of a tune almost as clearly as if I were actually listening to it") on a 5-point Likert scale ranging from 0 (certainly does not apply) to 4 (certainly applies). Although LSHS does not assess the broad range of potential NCP experiences (e.g., unusual thought content and suspiciousness), its items reliably capture low-level, subclinical symptoms on the psychosis spectrum [42]. Furthermore, the Brief Psychiatric Rating Scale (BPRS) [43], a clinical interview designed to track changes in psychotics symptoms, was administered to rule out frankly psychotic symptoms. Scores of 4 (moderate) or higher on the BPRS was an exclusion criterion, and all participants scored below that threshold. No participants in either group were excluded based on the BPRS scores. A scale of drug and alcohol use was used to assess the frequency of alcohol use, rated as 0 (no use) to 5 (almost daily) scale, i.e., higher scores represent more frequent use.

Postural Control and Working Memory in NCP

\section{Postural Control}

Postural sway data were acquired using an Advanced Mechanical Technology Incorporated (AMTI) AccuSway force platform (Watertown, MA, USA). Participants were instructed to stand still on the force platform with their arms resting at their sides while completing four 2-min-long task conditions: (1) eyes open, open base (EOOB); (2) eyes closed, open base (ECOB); (3) eyes open, closed base (EOCB); and (4) eyes closed, closed base (ECCB). The sway conditions are designed to provide increased challenge to cerebellar function by decreasing proprioceptive information (i.e., feet together) and sensory information (i.e., eyes closed). The center of pressure (COP) was the variable of interest, and it recorded at a sampling rate of $50 \mathrm{~Hz}$. We applied a 9th-order Butterworth filter with a $20-\mathrm{Hz}$ cut-off frequency to isolate the low-frequency postural sway process in the recorded data. COP and the $95 \%$ confidence interval of COP area were measured using principle component analysis [44]. Higher COP area is associated with poorer postural control. As noted, we predicted that deficits are subtler in the NCP population; we thus used the easiest condition with ample sensory cues (EOOB) and the most challenging condition with significantly decreased sensory cues (ECCB) in analyses.

\section{Cognitive Measures}

The study only included a limited cognitive battery, which focused on working memory. Trained assessors administered 2 tests from the Wechsler Memory Scale (WMS)-III [45]. In the Spatial Span task measuring visual working memory, participants were asked to tap cubes on a board of 10 irregularly spaced cubes in the same order as the administrator and then in reverse order. The Letter-Number Span task, measuring verbal working memory, is orally administered; the participants were asked to remember and reorder strings of letters and numbers and repeat them to the administrator. The age- and gender-corrected scores for each test were used in the analyses, and higher scores represent higher cognitive function.

\section{Statistical Approach}

Independent $t$ tests and $\chi^{2}$ tests were used to examine possible differences between the low- and high-NCP groups in demographic and clinical characteristics. For postural sway analyses, outliers were identified a priori as values $>3$ standard deviations from the group mean. Across both conditions, a total of 5 outliers were removed. Four data points were removed from the low-NCP group and 1 from the high-NCP group. Considering that alcohol use can impact cerebellar function [28,34], we controlled for frequency of alcohol use in all analyses. A $2 \times 2$ (condition $\times$ group) repeatedmeasures ANCOVA was conducted to examine group differences in postural sway. Post hoc univariate ANCOVAs were employed to further examine these differences. Lastly, partial correlations were used to test the predicted associations between both verbal and visual working memory and sway area in the high-NCP group. All analyses were conducted using two-tailed tests.

\section{Results}

Demographic, Clinical, and Cognitive Characteristic

The demographic, clinical, and cognitive characteristics are presented in Table 1. Independent sample $t$ tests 
Table 1. Demographic, clinical, and cognitive characteristics

\begin{tabular}{|c|c|c|c|c|}
\hline & $\begin{array}{l}\text { High-NCP } \\
\text { group }\end{array}$ & $\begin{array}{l}\text { Low-NCP } \\
\text { group }\end{array}$ & Total & $\begin{array}{l}\text { Group } \\
\text { differences }\end{array}$ \\
\hline \multicolumn{5}{|l|}{ Gender } \\
\hline Male & $17(45.9)$ & $16(51.6)$ & $33(48.5)$ & ns \\
\hline Female & $20(54.1)$ & $15(48.4)$ & $35(51.5)$ & \\
\hline Total & 37 & 31 & 68 & \\
\hline Age, years & $19.03 \pm 1.5$ & $19.23 \pm 1.56$ & $19.12 \pm 1.56$ & ns \\
\hline Parental education, years & $15.74 \pm 1.64$ & $15.01 \pm 3.45$ & $15.41 \pm 2.63$ & ns \\
\hline NCP symptoms ${ }^{\mathrm{a}}$ & $26.62 \pm 2.97$ & $1.81 \pm 1.05$ & $15.31 \pm 12.66$ & $p<0.001$ \\
\hline Letter-number span task score & $47.84 \pm 7.10$ & $47.84 \pm 7.50$ & $47.84 \pm 7.23$ & ns \\
\hline Spatial span task score & $46.59 \pm 9.27$ & $50.42 \pm 10.74$ & $48.34 \pm 10.08$ & ns \\
\hline
\end{tabular}

Values are expressed as $n(\%)$ or mean \pm SD. NCP, nonclinical psychosis.

a Total score on the Launay-Slade Hallucination Scale.

and $\chi^{2}$ tests revealed no significant differences in age $\left(t_{66}=0.52, p=0.60\right)$, gender $\left(\chi_{1}^{2}=0.22, p=0.64\right)$, and parental education $\left(t_{66}=-1.14, p=0.26\right)$ between the lowand high-NCP groups. Reflective of the sampling method, an independent sample $t$ test demonstrated that the high-NCP group (mean 26.62; SD 2.97) presented with significantly elevated NCP symptoms (i.e., sum scores on LSHS) when compared to the low-NCP group (mean $1.81, \mathrm{SD}=1.05)\left(t_{66}=-44.29, p<0.0001\right)$. Lastly, independent sample $t$ tests showed no group differences in the Letter-Number Span $\left(t_{66}=0, p=1\right)$ and Spatial Span $\left(t_{66}=1.58, p=0.12\right)$ tasks.

\section{Sway Area Differences}

A $2 \times 2$ repeated measures ANCOVA indicated a significant main effect of group $\left(F_{1,61}=4.87, p=0.03, \eta_{\mathrm{p}}^{2}=\right.$ $0.07)$ and main effect of balance condition $\left(F_{1,61}=40.22\right.$, $p<0.0001, \eta_{\mathrm{p}}^{2}=0.4$ ) (Fig. 1). Consistent with our prediction, a post hoc univariate ANCOVA revealed a significant main effect of group in the ECCB condition. The mean COP values in the high-NCP group were significantly elevated when compared to the low-NCP group $\left(F_{1,63}=5.63, p=0.02, \eta_{p}^{2}=0.08\right)$. However, the COP values were not significantly different in the EOOB condition $\left(F_{1,62}=2.88, p=0.1, \eta_{\mathrm{p}}^{2}=0.04\right)$ (Fig. 1).

\section{Associations between Sway Area and Cognition in High NCP}

The partial correlations revealed that, when controlling for alcohol use, larger sway area in the ECCB condition was associated with poorer scores in the Letter-Number Span task ( $r=-0.38, p=0.02)$ (Fig. 2) while the Spatial
Span task was not significantly correlated $(r=-0.26, p=$ $0.13)$. In the EOOB condition, however, when controlling for alcohol use, the Spatial Span task was associated with postural control $(r=-0.34, p=0.05)$ but the Letter-Number Span task was not $(r=-0.22, p=0.21)$.

\section{Discussion}

To our knowledge, this the first study to examine postural control and cognitive correlates in the NCP population. We found that high-NCP individuals presented with postural instability relative to the low-NCP controls. Notably, these findings are consistent with previously identified deficits in the same motor area in the CHR $[17,21]$ and schizophrenia $[27,32,36]$ populations. Furthermore, the degree of sway aberrations was correlated with poorer performance in the working-memory tasks. Taken together, these results provide further evidence of implications of CTCC circuit vulnerability across a continuum of psychosis. This is also in line with the cognitive dysmetria theory of psychosis, which posits that general dysfunction in sensorimotor and cognitive processes is associated with impairments in the CTCC network $[24,25]$.

This study provides additional support for aberrant postural control as a marker of motor-domain vulnerability, and studies examining schizophrenia and CHR populations show that balance control is largely affected. Research utilizing the same paradigm as in this study has revealed poorer postural control in these populations compared with controls across all conditions [17, 21,27]. In addition, given that postural control is heavily reliant 


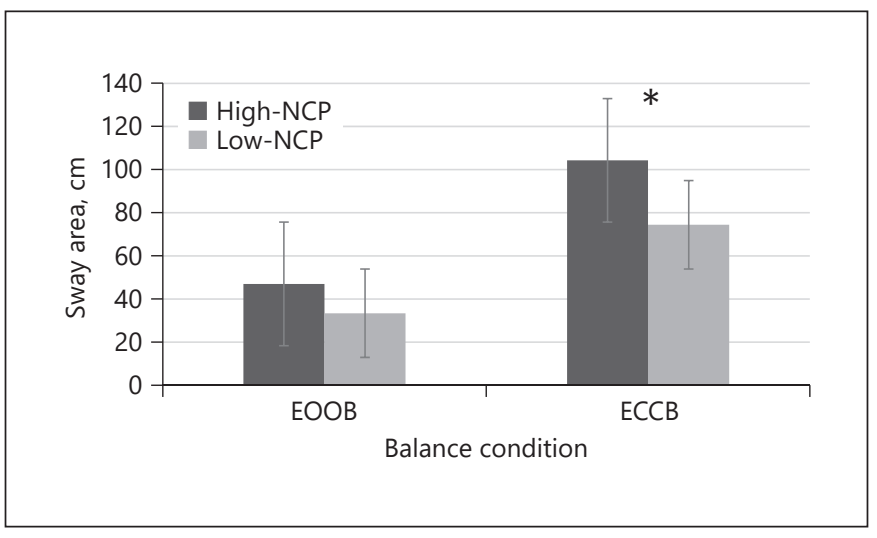

Fig. 1. Sway area for high-NCP participants and low-NCP controls across the 2 balance conditions. High-NCP participants showed significantly worse postural control (greater sway) in the most challenging condition. Error bars indicate the standard error of the mean. ${ }^{*} p<0.05$.

on sensory input [46] and that the cerebellum plays an important role in sensorimotor integration $[47,48]$, postural deficits also support dysregulation in this neurological domain. Thus far, this has been noted later in the course of an illness $[49,50]$ as well as in psychosis-prone healthy individuals [51]. In addition, findings from a recent study indicating that aberrations in postural sway are evident in the SPD population provided additional evidence for motor deficits and affected sensory integration across the psychosis continuum [36]. Notably, the authors found that SPD participants performed significantly worse than controls, and that their postural sway abnormalities were comparable to those of participants with schizophrenia. Considering that individuals with SPD do not present with extensive functional decline in most neurobehavioral domains seen in schizophrenia [52], these findings suggest that postural deficits may be a result of a distinct psychosis risk pathway. Our findings are in line with the abovementioned research and support the notion that sensory integration could be affected across the psychosis spectrum.

As previously noted, the current postural sway paradigm is a sensitive measure of cerebellar dysfunction, as postural control deficits are more evident when the challenge to the cerebellum is increased $[21,27,36]$. Our findings indicate that the high-NCP group exhibited deficits in postural sway in the condition that most significantly challenged the CTCC circuit (i.e., when both proprioceptive and visual cues were decreased) but not in the easier condition (where cues in both domains were provided). Considering that deficits are evident across both condi-

Postural Control and Working Memory in NCP

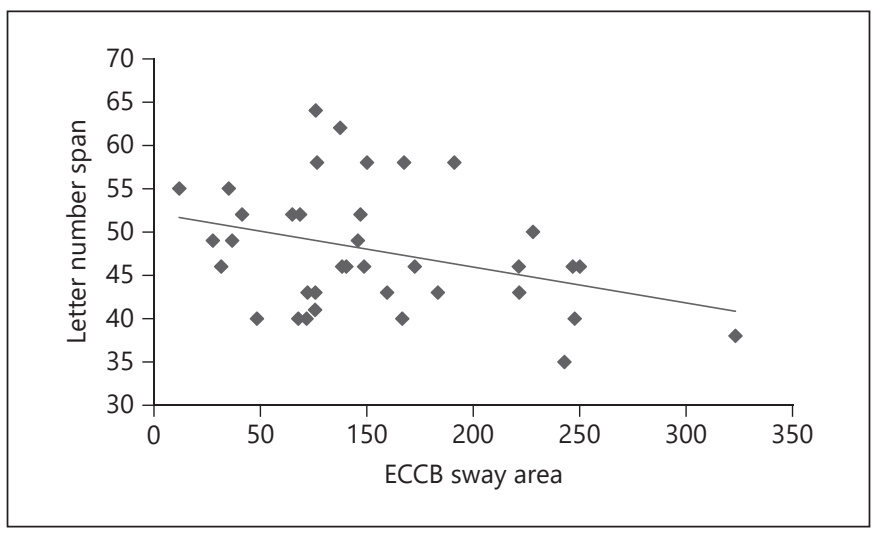

Fig. 2. Correlation between sway area in the most challenging condition (ECCB) and working memory in the high-NCP group.

tions in schizophrenia and CHR [17, 21, 27] but could only be detected in the more difficult one in NCP, it is likely that these aberrations change in severity across the psychosis continuum. Furthermore, this finding highlights the importance of advances in instrumental measures that can detect subtle deficits and provide more robust evidence, thereby improving our understanding of the motor domains implicated in the etiology of psychotic disorders [53].

In schizophrenia, converging evidence suggests that cerebellar dysfunction could account for some cognitive symptoms [54]. Indeed, ample research indicates that the dysfunction in the CTCC circuit is related to deficits in higher-order cognitive function including working memory $[38,55-58]$. Though research on this topic is limited, motor and working-memory correlates have been reported in schizophrenia populations [59].

In this study, in the most challenging condition, larger sway area was associated with poorer performance in the verbal working memory task; in the easier condition, it was associated with the visual working memory task. Evidence suggests that verbal and spatial processing are differently lateralized $[60,61]$, likely due to the modality of the stimulus [62]. Therefore, these findings could also be attributed to the different information processing domains involved in the tasks. Specifically, visual working memory relies on visual sensory input. Likewise, in the EOOB condition, participants can rely on visual input, and the research suggests that more infrequent visual feedback does not result in visuomotor activation in the cerebellum [63]. Conversely, the ECCB condition and 
verbal working memory do not rely on visual sensory input, and they could be affected by different cerebellar processes within the cerebellum and across a broad array of neural networks. Additionally, a recent meta-analysis revealed altered cerebellar topography in schizophrenia patients, particularly that related to working memory [38]. Therefore, it could be that different cerebellar regions are not affected significantly enough in the NCP to cause more widespread deficits. Of note, our results indicate that the 2 groups did not differ in either working memory domain, and the observed deficits were only relative to postural control; therefore, these findings should be interpreted with some caution.

Though implicated on a subtler level in the NCP group, postural sway aberrations and the cognitive correlates support the emerging evidence of the cerebellar dysfunction as implicated in the development and progression of psychosis. Overall, our study provides further evidence of the role of the CTCC circuit in the pathogenesis of psychosis. The observed deficits are consistent with the cognitive dysmetria theory, in that the potentially impacted cerebellar networks may be contributing to the postural sway deficits and the cognitive correlates [24, 25]. Therefore, while it is well-established that this theory is a valuable framework for explaining schizophrenia, it may also be useful for trying to understand the subtler deficits observed in the NCP population.

Lastly, this study presented with both strengths and limitations. The advantage of the study was the utilization of an instrumental measure of postural control which provided more reliable and discrete values than observerrated measures [64]. However, although postural sway has been shown to be a sensitive measure of cerebellar dysfunction [17, 21, 27, 34], we did not directly measure brain abnormalities. Future research would benefit from investigating deficits in the CTCC circuit in the NCP population using neuroimaging approaches. Furthermore, although we controlled for alcohol use in all analyses, further examination of such influences in the NCP population is warranted, considering that alcohol use significantly moderates sway aberrations in individuals with schizophrenia [28, 34]. In addition, our cognitive battery was limited to the verbal and visual working memory. Considering that other studies have found that cognition is affected and associated with psychotic-like symptoms in NCP individuals [10] and continues to be impacted in CHR states, and that later conversion to a psychotic disorder is associated with more severe dysfunction [65], additional examination of cognitive function is crucial. Future studies could benefit from utilizing measures of dif- ferent cognitive domains to establish whether cognition (in a broader sense) is impacted in the NCP population. Finally, although NCP experiences are related to an increased risk for transitioning to a formal psychotic disorder such as schizophrenia [5-8], only a small portion of these individuals will experience a psychotic outcome. Future work that longitudinally examines the progression of motor abnormalities could improve our understanding of their predictive value and role as a distinctive domain contributing to the onset of psychosis.

Our findings are largely consistent with what has been observed in CHR, SPD, and schizophrenia populations, indicating a shared vulnerability. Considering that subtle motor deficits can be detected well before the onset of psychosis, investigating them in the NCP population could provide critical insights into the progression of the noted abnormalities and their contribution to the onset of psychosis.

\section{Statement of Ethics}

Subjects gave their written informed consent. The study protocol was approved by the Institutional Review Board on human research.

\section{Disclosure Statement}

Authors have no conflicts of interest to report.

\section{Funding Sources}

This study was supported by National Institutes of Mental Health grant R01MH094650 awarded to Dr. Mittal.

\section{Author Contributions}

Ms. Ristanovic conceptualized the study, conducted the analyses, and drafted the manuscript under Dr. Mittal's supervision. Mr. Osborne, Ms. Vargas, and Ms. Gupta helped with data analyses, interpreting the results, and drafting the manuscript. Dr. Mittal attained the funding and provided critical revisions to the manuscript. 


\section{References}

1 Kelleher I, Cannon M. Psychotic-like experiences in the general population: characterizing a high-risk group for psychosis. Psychol Med. 2011 Jan;41(1):1-6.

2 van Os J, Hanssen M, Bijl RV, Ravelli A. Strauss (1969) revisited: a psychosis continuum in the general population? Schizophr Res. 2000 Sep;45(1-2):11-20.

3 Verdoux H, van Os J. Psychotic symptoms in non-clinical populations and the continuum of psychosis. Schizophr Res. 2002 Mar;54(12):59-65.

4 Dolphin L, Dooley B, Fitzgerald A. Prevalence and correlates of psychotic like experiences in a nationally representative community sample of adolescents in Ireland. Schizophr Res. 2015 Dec;169(1-3):241-7.

5 Zammit S, Odd D, Horwood J, Thompson A, Thomas K, Menezes $\mathrm{P}$, et al. Investigating whether adverse prenatal and perinatal events are associated with non-clinical psychotic symptoms at age 12 years in the ALSPAC birth cohort. Psychol Med. 2009 Sep;39(9): 1457-67.

6 Kaymaz N, Drukker M, Lieb R, Wittchen HU, Werbeloff N, Weiser M, et al. Do subthreshold psychotic experiences predict clinical outcomes in unselected non-help-seeking population-based samples? A systematic review and meta-analysis, enriched with new results. Psychol Med. 2012 Nov;42(11):2239-53.

7 Dominguez MD, Saka MC, Lieb R, Wittchen HU, van Os J. Early expression of negative/ disorganized symptoms predicting psychotic experiences and subsequent clinical psychosis: a 10-year study. Am J Psychiatry. 2010 Sep;167(9):1075-82.

8 Dominguez MD, Wichers M, Lieb R, Wittchen HU, van Os J. Evidence that onset of clinical psychosis is an outcome of progressively more persistent subclinical psychotic experiences: an 8-year cohort study. Schizophr Bull. 2011 Jan;37(1):84-93.

9 Barkus E, Stirling J, Hopkins R, McKie S, Lewis $\mathrm{S}$. Cognitive and neural processes in nonclinical auditory hallucinations. Br J Psychiatry Suppl. 2007 Dec;51 S51:s76-81.

10 Horwood J, Salvi G, Thomas K, Duffy L, Gunnell D, Hollis C, et al. IQ and non-clinical psychotic symptoms in 12-year-olds: results from the ALSPAC birth cohort. Br J Psychiatry. 2008 Sep;193(3):185-91.

11 Mittal VA, Orr JM, Pelletier A, Dean DJ, Smith A, Lunsford-Avery J. Hypothalamicpituitary-adrenal axis dysfunction in nonclinical psychosis. Psychiatry Res. 2013 Apr; 206(2-3):315-7.

12 Mittal VA, Smolen A, Dean DJ, Pelletier AL, Lunsford-Avery J, Smith A. BDNF Val66Met and spontaneous dyskinesias in non-clinical psychosis. Schizophr Res. 2012 Sep;140(1-3): 65-70.

Postural Control and Working Memory in NCP
13 Hirjak D, Thomann PA, Kubera KM, Wolf ND, Sambataro F, Wolf RC. Motor dysfunction within the schizophrenia-spectrum: A dimensional step towards an underappreciated domain. Schizophr Res. 2015 Dec;169(13):217-33.

14 Compton MT, Fantes F, Wan CR, Johnson S, Walker EF. Abnormal movements in first-episode, nonaffective psychosis: dyskinesias, stereotypies, and catatonic-like signs. Psychiatry Res. 2015 Mar;226(1):192-7.

15 Walther S, Strik W. Motor symptoms and schizophrenia. Neuropsychobiology. 2012; 66(2):77-92.

16 Bernard JA, Mittal VA. Cerebellar-motor dysfunction in schizophrenia and psychosisrisk: the importance of regional cerebellar analysis approaches. Front Psychiatry. 2014 Nov; $5: 160$

17 Dean DJ, Kent JS, Bernard JA, Orr JM, Gupta T, Pelletier-Baldelli A, et al. Increased postural sway predicts negative symptom progression in youth at ultrahigh risk for psychosis. Schizophr Res. 2015 Mar;162(1-3):86-9.

18 Mittal VA, Orr JM, Turner JA, Pelletier AL, Dean DJ, Lunsford-Avery J, et al. Striatal abnormalities and spontaneous dyskinesias in non-clinical psychosis. Schizophr Res. 2013 Dec;151(1-3):141-7.

19 Teng YL, Chen CL, Lou SZ, Wang WT, Wu JY, Ma HI, et al. Postural Stability of Patients with Schizophrenia during Challenging Sensory Conditions: Implication of Sensory Integration for Postural Control. PLoS One. 2016 Jun;11(6):e0158219.

20 Willems AE, Sommer IE, Tenback DE, Koning JP, van Harten PN. Instrumental measurements of spontaneous dyskinesia and schizotypy in subjects with auditory verbal hallucinations and healthy controls. Psychiatry Res. 2016 Oct;244:24-7.

21 Bernard JA, Dean DJ, Kent JS, Orr JM, Pelletier-Baldelli A, Lunsford-Avery JR, et al. Cerebellar networks in individuals at ultra highrisk of psychosis: impact on postural sway and symptom severity. Hum Brain Mapp. 2014 Aug;35(8):4064-78.

22 Barch DM. Cerebellar-thalamic connectivity in schizophrenia. Schizophr Bull. 2014 Nov; 40(6):1200-3.

23 Walther S. Psychomotor symptoms of schizophrenia map on the cerebral motor circuit. Psychiatry Res. 2015 Sep;233(3):293-8.

24 Andreasen NC, Paradiso S, O'Leary DS. "Cognitive dysmetria" as an integrative theory of schizophrenia: a dysfunction in corticalsubcortical-cerebellar circuitry? Schizophr Bull. 1998;24(2):203-18.

25 Andreasen NC, Pierson R. The role of the cerebellum in schizophrenia. Biol Psychiatry. 2008 Jul;64(2):81-8.

26 Osborne KJ, Bernard JA, Gupta T, Dean DJ, Millman Z, Vargas T, et al. Beat gestures and postural control in youth at ultrahigh risk for psychosis. Schizophr Res. 2017 Jul; 185:197-9.
27 Kent JS, Hong SL, Bolbecker AR, Klaunig MJ, Forsyth JK, O'Donnell BF, et al. Motor deficits in schizophrenia quantified by nonlinear analysis of postural sway. PLoS One. 2012; 7(8):e41808.

28 Sullivan EV, Rosenbloom MJ, Pfefferbaum A. Balance and gait deficits in schizophrenia compounded by the comorbidity of alcoholism. Am J Psychiatry. 2004 Apr;161(4):751-5.

29 Coesmans M, Röder CH, Smit AE, Koekkoek SK, De Zeeuw CI, Frens MA, et al. Cerebellar motor learning deficits in medicated and medication-free men with recent-onset schizophrenia. J Psychiatry Neurosci. 2014 Jan;39(1):E3-11.

30 Pedersen A, Siegmund A, Ohrmann P, Rist F, Rothermundt M, Suslow T, et al. Reduced implicit and explicit sequence learning in firstepisode schizophrenia. Neuropsychologia. 2008 Jan;46(1):186-95.

31 Bolbecker AR, Hetrick WP, Johannesen JK, O'Donnell BF, Steinmetz JE, Shekhar AS. Secretin effects on cerebellar-dependent motor learning in schizophrenia. Am J Psychiatry. 2009 Apr;166(4):460-6.

32 Marvel CL, Schwartz BL, Rosse RB. A quantitative measure of postural sway deficits in schizophrenia. Schizophr Res. 2004 Jun;68(23):363-72.

33 Gupta T, Dean DJ, Kelley NJ, Bernard JA, Ristanovic I, Mittal VA. Cerebellar Transcranial Direct Current Stimulation Improves Procedural Learning in Nonclinical Psychosis: A Double-Blind Crossover Study. Schizophr Bull. 2018 Oct;44(6):1373-80.

34 Deshmukh A, Rosenbloom MJ, Pfefferbaum A, Sullivan EV. Clinical signs of cerebellar dysfunction in schizophrenia, alcoholism, and their comorbidity. Schizophr Res. 2002 Oct;57(2-3):281-91.

35 Morton SM, Bastian AJ. Cerebellar control of balance and locomotion. Neuroscientist. 2004 Jun;10(3):247-59.

36 Apthorp D, Bolbecker AR, Bartolomeo LA, O'Donnell BF, Hetrick WP. Postural Sway Abnormalities in Schizotypal Personality Disorder. Schizophr Bull. 2018 Oct. https://doi. org/10.1093/schbul/sby141.

37 Bernard JA, Orr JM, Dean DJ, Mittal VA. The cerebellum and learning of non-motor associations in individuals at clinical-high risk for psychosis. Neuroimage Clin. 2018 Mar; 19: 137-46.

38 Bernard JA, Mittal VA. Dysfunctional Activation of the Cerebellum in Schizophrenia: A Functional Neuroimaging Meta-Analysis. Clin Psychol Sci. 2015 Jul;3(4):545-66.

39 Bentall RP, Slade PD. Reliability of a scale measuring disposition towards hallucination: a brief report. Pers Individ Dif. 1985;6(4): 527-9.

40 Johns LC. Hallucinations in the general population. Curr Psychiatry Rep. 2005 Jun;7(3): 162-7. 
41 Vellante M, Larøi F, Cella M, Raballo A, Petretto DR, Preti A. Hallucination-like experiences in the nonclinical population. J Nerv Ment Dis. 2012 Apr;200(4):310-5.

42 Serper M, Dill CA, Chang N, Kot T, Elliot J. Factorial structure of the hallucinatory experience: continuity of experience in psychotic and normal individuals. J Nerv Ment Dis. 2005 Apr;193(4):265-72.

43 Overall JE, Gorham DR. The Brief Psychiatric Rating-Scale. Psychol Rep. 1962;10(3):799812.

44 Oliveira LF, Simpson DM, Nadal J. Calculation of area of stabilometric signals using principal component analysis. Physiol Meas. 1996 Nov; 17(4):305-12.

45 Wechsler D. Wechsler memory scale. San Antonio, TX, US: Psychological Corporation; 1945.

46 Kuo AD, Speers RA, Peterka RJ, Horak FB. Effect of altered sensory conditions on multivariate descriptors of human postural sway. Exp Brain Res. 1998 Sep;122(2):185-95.

47 Bastian AJ. Learning to predict the future: the cerebellum adapts feedforward movement control. Curr Opin Neurobiol. 2006 Dec; 16(6):645-9.

48 Wolpert DM, Miall RC, Kawato M. Internal models in the cerebellum. Trends Cogn Sci. 1998 Sep;2(9):338-47.

49 Mittal VA, Dean DJ, Bernard JA, Orr JM, Pelletier-Baldelli A, Carol EE, et al. Neurological soft signs predict abnormal cerebellar-thalamic tract development and negative symptoms in adolescents at high risk for psychosis: a longitudinal perspective. Schizophr Bull. 2014 Nov;40(6):1204-15.
50 Dazzan P, Murray RM. Neurological soft signs in first-episode psychosis: a systematic review. Br J Psychiatry Suppl. 2002 Sep;43 S43:s50-7.

51 Barkus E, Stirling J, Hopkins R, Lewis S. The presence of neurological soft signs along the psychosis proneness continuum. Schizophr Bull. 2006 Jul;32(3):573-7.

52 Rosell DR, Futterman SE, McMaster A, Siever LJ. Schizotypal personality disorder: a current review. Curr Psychiatry Rep. 2014 Jul;16(7): 452.

53 van Harten PN, Walther S, Kent JS, Sponheim SR, Mittal VA. The clinical and prognostic value of motor abnormalities in psychosis, and the importance of instrumental assessment. Neurosci Biobehav Rev. 2017 Sep;80: 476-87.

54 Picard H, Amado I, Mouchet-Mages S, Olié JP, Krebs MO. The role of the cerebellum in schizophrenia: an update of clinical, cognitive, and functional evidences. Schizophr Bull. 2008 Jan;34(1):155-72.

55 Stoodley CJ. The cerebellum and cognition: evidence from functional imaging studies. Cerebellum. 2012 Jun;11(2):352-65.

56 Mendrek A, Kiehl KA, Smith AM, Irwin D, Forster BB, Liddle PF. Dysfunction of a distributed neural circuitry in schizophrenia patients during a working-memory performance. Psychol Med. 2005 Feb;35(2):187-96.

57 Wiser AK, Andreasen NC, O'Leary DS, Watkins GL, Boles Ponto LL, Hichwa RD. Dysfunctional cortico-cerebellar circuits cause 'cognitive dysmetria' in schizophrenia. Neuroreport. 1998 Jun;9(8):1895-9.
58 Pauly K, Seiferth NY, Kellermann T, Ruhrmann S, Daumann B, Backes V, et al. The interaction of working memory and emotion in persons clinically at risk for psychosis: an fMRI pilot study. Schizophr Res. 2010 Jul;120(1-3):167-76.

59 Silver H, Feldman P, Bilker W, Gur RC. Working memory deficit as a core neuropsychological dysfunction in schizophrenia. Am J Psychiatry. 2003 Oct;160(10):1809-16.

60 Stoodley CJ, Valera EM, Schmahmann JD. Functional topography of the cerebellum for motor and cognitive tasks: an fMRI study. Neuroimage. 2012 Jan;59(2):1560-70.

61 Stoodley CJ, Schmahmann JD. Functional topography in the human cerebellum: a metaanalysis of neuroimaging studies. Neuroimage. 2009 Jan;44(2):489-501.

62 Marvel CL, Desmond JE. Functional topography of the cerebellum in verbal working memory. Neuropsychol Rev. 2010 Sep;20(3): 271-9.

63 Vaillancourt DE, Mayka MA, Corcos DM. Intermittent visuomotor processing in the human cerebellum, parietal cortex, and premotor cortex. J Neurophysiol. 2006 Feb;95(2): $922-31$.

64 Docx L, Sabbe B, Fransen E, Bervoets C, Hulstijn W, Van Den Bossche MJ, et al. Longitudinal evaluation of the psychomotor syndrome in schizophrenia. J Neuropsychiatry Clin Neurosci. 2014;26(4):359-68.

65 Bora E, Lin A, Wood SJ, Yung AR, McGorry PD, Pantelis C. Cognitive deficits in youth with familial and clinical high risk to psychosis: a systematic review and meta-analysis. Acta Psychiatr Scand. 2014 Jul;130(1):1-15. 